\title{
Characterization of pancreatic ductal adenocarcinoma using whole transcriptome sequencing and copy number analysis by single-nucleotide polymorphism array
}

\author{
MARIACRISTINA DI MARCO ${ }^{1}$, ANNALISA ASTOLFI ${ }^{2}$, ELISA GRASSI ${ }^{1}$, SILVIA VECCHIARELLI ${ }^{1}$, \\ MARINA MACCHINI $^{1}$, VALENTINA INDIO ${ }^{2}$, RICCARDO CASADEI $^{3}$, CLAUDIO RICCI $^{3}$, MARIELDA D'AMBRA $^{3}$, \\ GIOVANNI TAFFURELLI ${ }^{3}$, CARLA SERRA ${ }^{4}$, GIORGIO ERCOLANI ${ }^{5}$, DONATELLA SANTINI ${ }^{6}$, \\ ANTONIA D'ERRICO ${ }^{1}$, ANTONIO DANIELE PINNA ${ }^{3,6}$, FRANCESCO MINNI ${ }^{3}$, \\ SANDRA DURANTE $^{2}$, LAURA RAFFAELLA MARTELLA ${ }^{1}$ and GUIDO BIASCO ${ }^{1,2}$

\footnotetext{
${ }^{1}$ Department of Experimental, Diagnostic and Specialty Medicine; ${ }^{2}$ Interdepartmental Center of Cancer Research; Departments of ${ }^{3}$ Medical and Surgical Sciences and ${ }^{4}$ Digestive Diseases and Internal Medicine;

${ }^{5}$ Liver and Multiorgan Transplant Unit; ${ }^{6}$ Pathology Unit, Sant'Orsola-Malpighi Hospital, Bologna I-40100, Italy
}

Received March 13, 2015; Accepted August 27, 2015

DOI: $10.3892 / \mathrm{mmr} .2015 .4344$

\begin{abstract}
The aim of the current study was to implement whole transcriptome massively parallel sequencing (RNASeq) and copy number analysis to investigate the molecular biology of pancreatic ductal adenocarcinoma (PDAC). Samples from 16 patients with PDAC were collected by ultrasound-guided biopsy or from surgical specimens for DNA and RNA extraction. All samples were analyzed by RNASeq performed at $75 \times 2$ base pairs on a HiScanSQ Illumina platform. Single-nucleotide variants (SNVs) were detected with SNVMix and filtered on dbSNP, 1000 Genomes and Cosmic. Non-synonymous SNVs were analyzed with SNPs\&GO and PROVEAN. A total of 13 samples were analyzed by high resolution copy number analysis on an Affymetrix SNP array 6.0. RNAseq resulted in an average of 264 coding non-synonymous novel SNVs (ranging from 146-374) and 16 novel insertions or deletions (In/Dels) (ranging from 6-24) for each sample, of which a mean of $11.2 \%$ were disease-associated and somatic events, while $34.7 \%$ were frameshift somatic In/Dels. From this analysis, alterations in the known oncogenes associated with PDAC were observed, including Kirsten rat sarcoma viral oncogene homolog (KRAS) mutations (93.7\%) and inactivation of cyclin-dependent kinase inhibitor 2A (CDKN2A) (50\%), mothers against decapentaplegic homolog 4 (SMAD4) $(50 \%)$, and tumor protein 53 (TP53) (56\%). One case that was negative for KRAS exhibited a G13D neuroblastoma
\end{abstract}

Correspondence to: Dr Elisa Grassi, Department of Experimental, Diagnostic and Specialty Medicine, Sant'Orsola-Malpighi Hospital, 11 Massarenti Street, Bologna I-40100, Italy

E-mail: elisa.grax@gmail.com

Key words: pancreatic cancer, whole transcriptome sequencing, copy number analysis
RAS viral oncogene homolog mutation. In addition, gene fusions were detected in 10 samples for a total of 23 different intra- or inter-chromosomal rearrangements, however, a recurrent fusion transcript remains to be identified. SNP arrays identified macroscopic and cryptic cytogenetic alterations in $85 \%$ of patients. Gains were observed in the chromosome arms $6 \mathrm{p}, 12 \mathrm{p}, 18 \mathrm{q}$ and $19 \mathrm{q}$ which contain KRAS, GATA binding protein 6 , protein kinase B and cyclin D3. Deletions were identified on chromosome arms 1p, 9p, 6p, 18q, 10q, $15 q, 17 p, 21 q$ and $19 q$ which involve TP53, CDKN2A/B, SMAD4, runt-related transcription factor 2, AT-rich interactive domain-containing protein $1 \mathrm{~A}$, phosphatase and tensin homolog and serine/threonine kinase 11. In conclusion, genetic alterations in PDCA were observed to involve numerous pathways including cell migration, transforming growth factor- $\beta$ signaling, apoptosis, cell proliferation and DNA damage repair. However, signaling alterations were not observed in all tumors and key mutations appeared to differ between PDAC cases.

\section{Introduction}

Pancreatic ductal adenocarcinoma (PDAC) is the fourth leading cause of cancer-associated mortality, with a five-year survival rate of $5 \%$. Surgery remains the most effective treatment, however only $20 \%$ of patients are suitable for radical resection (1).

Advances in chemotherapy, such as FOLFIRINOX and gemcitabine plus nab-paclitaxel regimens, have resulted in an improvement in outcomes, and gemcitabine-based chemotherapy remains the gold-standard treatment, particularly in metastatic disease (2-4).

Despite limited impact on patient care, recent PDAC genomic characterization via the molecular dissection of somatic alterations has generated informative data on different types of cancer. However, advances in the development of novel PDAC therapeutic and early-detection strategies remain 
to be identified. The current study aimed to implement whole transcriptome massively parallel sequencing (RNASeq) and copy number analysis to investigate the molecular biology of PDAC, to aid in improving diagnosis and indicating potential targets for personalized diagnostic and therapeutic intervention. RNASeq is a powerful tool for the identification of cancer mutations underlying pancreatic carcinogenesis, and is an efficient approach for the detection of somatic events such as nucleotide substitution mutations and gene translocations with high resolution, via sequencing of the expressed gene (cell transcriptomes). In addition, this technology has the advantage of not being limited to known genes and additionally may detect novel transcripts and alternative splice forms (5).

The present study combined RNASeq and copy number analysis by the single-nucleotide polymorphism (SNP) array to obtain a comprehensive overview of genetic alterations in pancreatic cancer.

\section{Patients and methods}

The current study was conducted according to the principles of the Declaration of Helsinki, and written informed consent was obtained from all participants. The study was previously approved by the Independent Ethics Committee of Sant'Orsola-Malpighi Hospital (Bologna, Italy).

Sample collection and patient characteristics. A total of 24 PDAC samples were collected from ultrasound-guided biopsies or surgical specimens for DNA and RNA extraction, however 16 specimens were analyzed due to the exclusion of 3 cases for non-PDAC histology, 2 cases for insufficient nucleic acid extraction and 3 cases for estimated low cellularity. Patient characteristics are presented in Table I. The tissue samples were collected in cryogenic tubes (Ambion Life Technologies, Carlsbad, CA, USA) and stored at $-20^{\circ} \mathrm{C}$ in RNAlater solution (Ambion Life Technologies). Nucleic acid extraction was performed with the AllPrep RNA/DNA kit (Qiagen, Inc., Valencia, CA, USA) for tumor biopsies and the QIAAmp DNA Mini kit (Qiagen, Inc.) for peripheral blood DNA.

Sample cellularity. PDAC is characterized by a small quantity of adenocarcinoma cells (6). Evaluation of tumor cells in the sample was based on the presence and relative enrichment of the Kirsten rat sarcoma viral oncogene homolog (KRAS) mutation. The percentage of tumor cells in the clinical specimen was estimated using KRAS mutation Sanger sequencing. Samples with $10 \%$ more tumor alleles than normal alleles were included in the present study.

Copy number alteration analysis. Tumor DNA was labeled and hybridized to GeneChip SNP 6.0 arrays (Affymetrix, Inc., Santa Clara, CA, USA) following the manufacturer's instructions. Copy number analysis was conducted using Partek Genomic Suite software (version 6.5; Partek, Inc., St. Louis, MO, USA), using the segmentation algorithm and setting the parameters to identify large and cryptic regions of copy number alterations $(\mathrm{P}$-value $=0.001$; number of markers $=10$; signal to noise ratio $=0.3$ ). The copy number was assessed by comparing the intensity distribution to a reference set consisting of approximately 270 normal samples from individuals of different ethnicities derived from the HapMap database (http://hapmap.ncbi.nlm.nih.gov/).

Whole transcriptome massivelyparallel sequencing. Following RNA extraction using the AllPrep RNA/DNA kit (Qiagen, Inc.,), whole transcriptome sequencing was performed on the Illumina HiScanSQ platform, (Illumina, Inc., San Diego, CA, USA) using Illumina paired-end massively parallel sequencing in accordance with the manufacturer's instructions. Poly(A)-RNA was purified from 250-500 ng total RNA using poly-T oligo-attached magnetic beads (Illumina, Inc.), and libraries of cDNA fragments were sequenced at 2x80 base pairs (bp) read length in paired end mode. An average of 98.2 million reads/sample were produced.

For exome sequencing, genomic DNA from peripheral blood was fragmented, tagged, indexed and amplified with the Nextera Exome Enrichment kit (Illumina, Inc.) to an average library size of $350 \mathrm{bp}$. DNA libraries were hybridized to biotin-labeled 95-mer probes (Illumina, Inc.) designed to enrich $62 \mathrm{Mb}$ of genomic DNA covering more than 200,000 exons including exon-flanking regions. Sequencing was performed at $100 \mathrm{bp}$ in the paired end, producing on average 2.6 Gb per sample.

For bioinformatics analysis, the short reads were processed, quality-filtered and mapped on the human reference genome to identify all detectable variations in the sample, including single-nucleotide variants (SNVs), insertions or deletions (In/Dels) and large chromosomal rearrangements. Open-source software [BowTie (http://bowtie-bio. sourceforge.net/index.shtml), TopHat (https://ccb.jhu. edu/software/tophat/index.shtml), SAMtools (http://samtools. sourceforge.net/) and SNVMix (http://compbio.bccrc.ca/software/snvmix/)] was used for mapping. Novel variants were identified by comparison with public databases on human variability [1000 Genomes (http://www.1000genomes. org/), dbSNP (http://www.ncbi.nlm.nih.gov/SNP/) and Cosmic (http://cancer.sanger.ac.uk/cosmic)] and the potential effect of the non-synonymous SNVs was evaluated at a protein level with computational tools including SNPs\&GO (http://snps-and-go.biocomp.unibo.it/snps-and-go/),

SIFT (http://sift.jcvi.org/) and PolyPhen (http://genetics. bwh.harvard.edu/pph2/). deFuse (http://compbio.bccrc. ca/software/defuse/), ChimeraScan (https://code.google. com/p/chimerascan/) and FusionMap (http://www.arrayserver.com/wiki/index.php?title=FusionMap) were used to identify chromosomal rearrangements resulting in gene fusion.

Sanger sequencing. SNVs were confirmed by Sanger sequencing on tumor samples and peripheral blood DNA (when available) on an ABI 3730 Genetic Analyzer (Applied Biosystems Life Technologies, Monza, Italy). Primer pairs, designed with Primer Express software, version 3.0 (Applied Biosystems Life Technologies), were specific to amplify exons and the flanking intronic regions. PCR products were purified with the QIAquick PCR purification kit (Qiagen, Inc.) and sequenced on both strands using the BigDye Terminator v1.1 Cycle Sequencing kit (Applied Biosystems Life Technologies). 
Table I. Characteristics of the patient cohort with a median age of 65.5 years (range, 34-87).

\begin{tabular}{lc}
\hline Characteristic & \% of study population \\
\hline Gender & 23.5 \\
Male & 76.5 \\
Female & \\
Site of specimen & 94 \\
Pancreatic tumor & 6 \\
Hepatic metastasis & \\
Stage & 41 \\
I+II & 29.5 \\
III-LA & 29.5 \\
IV & \\
\hline
\end{tabular}

\section{Results}

Whole transcriptome sequencing of the 16 samples derived from surgical specimens or ultrasound-guided biopsies was conducted. Of these, 13 specimens additionally underwent copy number analysis using Affymetrix technology to obtain a comprehensive overview of the genetic alterations in pancreatic cancer.

SNP array identified focal or macroscopic amplifications and deletions in $85 \%$ of patients (Fig. 1). Gains frequently involved chromosome arms $12 \mathrm{p}, 18 \mathrm{q}, 19 \mathrm{q}$ and $6 \mathrm{p}$, which contain the oncogenes KRAS, GATA binding protein 6 (GATA6), protein kinase B (AKT2) and cyclin D3 (CCND3). Focal high copy number amplifications were identified in the $8 \mathrm{q} 24$ region involving the MYC gene, and in $17 q 12$ surrounding human epidermal growth factor receptor 2 (ERBB2) in one and two patients, respectively. Deletions were observed in greater than $70 \%$ of patients on chromosome arms 1p, 9p, 6p, 18q, 10q, 15q, 17p, 21q and 19p. Deleted genes were commonly those previously associated with PDAC, including tumor protein 53 (TP53), cyclin-dependent kinase inhibitor 2A (CDKN2A/B) and mothers against decapentaplegic homolog 4 (SMAD4). In addition, runt-related transcription factor 2, AT-rich interactive domain-containing protein 1A (ARID1A), phosphatase and tensin homolog (PTEN)and serine/threonine kinase 11 were identified to be deleted. An additional recurrent deletion involved chromosome $15 \mathrm{q}$, however a specific target oncosuppressor has not been identified in this region at present.

RNASeq identified an average of 264 coding non-synonymous novel SNVs (ranging from 146-374) and 16 novel In/Dels (ranging from 6-24) for each sample, of which a mean of $11.2 \%$ (from $4.8-17.6 \%$ ) were disease-associated and somatic events and $34.7 \%$ (from 13.3-52.2\%) were frameshift somatic In/Dels (for the patient with matched-normal DNA available). Furthermore, gene fusions were detected in 10 patients with a total of 23 different intra- or inter-chromosomal rearrangements, however the recurrent fusion transcript was not identified. The majority of rearrangements were inter-chromosomal $(66 \%)$ and did not alter the reading-frame $(51 \%)$.
KRAS was reported to have the greatest prevalence of somatic mutations (93.7\% of cases) and the mutations affected the known hotspot at codon 12 (G12D in 8 patients, G12V and G12R in 5 and 2 patients respectively) (7). In one case which was negative for KRAS, a G13D neuroblastoma RAS viral oncogene homolog (NRAS) mutation was identified. The prevalence of the RAS mutation in the current study is biased due to the experimental design, as samples were only included in the study if a mutation in a RAS gene was present. This strategy was designed to take into account the common occurrence of stromal desmoplasia in the majority of cases of PDAC that may affect the percentage of tumor cells present in biopsies or surgical specimens undergoing analysis. The percentage of tumor cells in the sample were estimated by quantifying the RAS mutation in the DNA, taking into account copy number gains or the loss of chromosome 12 . This analysis enabled the inclusion of 16 patients (out of the 20 initially assayed) that exhibited an average of $41.9 \pm 7.48 \%$ tumor cells in the sample.

The most recurrent genes observed to be altered by point mutations, small In/Dels and heterozygous or homozygous copy number loss are presented in Fig. 2, with TP53, CDKN2A and SMAD4 observed to be commonly inactivated genes. TP53 was inactivated in $56 \%$ of tumor samples due to somatic point mutations (5/16) or allelic loss (5/16), while CDKN2A function was disrupted in 8 of the 16 samples by missense or nonsense mutations and hetero/homozygous genomic loss. TP53 and CDKN2A alterations were frequently coupled, with 6 of the 11 patients exhibiting mutations in these two genes. Transforming growth factor- $\beta$ (TGF- $\beta$ ) signaling was impaired in $50 \%$ of patients, frequently due to hetero- or homozygous focal loss of the SMAD4 locus. In addition, SMAD3 was frequently inactivated by point mutation, gene fusion or allelic loss. Somatic mutations in the additional genes identified had reduced ocurrence, compared with the frequency of KRAS, SMAD4, TP53 and CDKN2A. However, an exception was ARID1A, the inactivation of which was observed in 6 of the 16 patients due to heterozygous focal genomic deletions or frameshift small In/Dels. In addition, the known oncosuppressor gene, PTEN, was inactivated due to nonsense or missense mutations, or chromosome arm deletion. Notably, PTEN alterations were more frequent in patients with advanced disease (4/8) compared with early stage subjects $(0 / 7)$.

The DNA damage response pathway is significantly impaired in pancreatic tumorigenesis (8). Pathway analysis of the genes carrying somatic alterations in a minimum of two patients exhibted a significant enrichment in DNA repair mechanisms $(\mathrm{P}=0.007$, fold enrichment $=9.6)$. Of note, mismatch repair, base-excision and nucleotide excision repair pathways were altered in different patients. In Fig. 2 the most recurrent genes altered by point mutations, small In/Dels and heterozygous or homozygous copy number loss are presented.

\section{Discussion}

Pancreatic cancer presents genetic heterogeneity with a high number of mutations occurring in single cases. Four genes with a high prevalence of mutations have been identified: KRAS mutation, CDKN2A inactivation (considered to be early events in the PDAC progression model), TP53 and SMAD4 inactivation (considered as later events) $(9,10)$. 


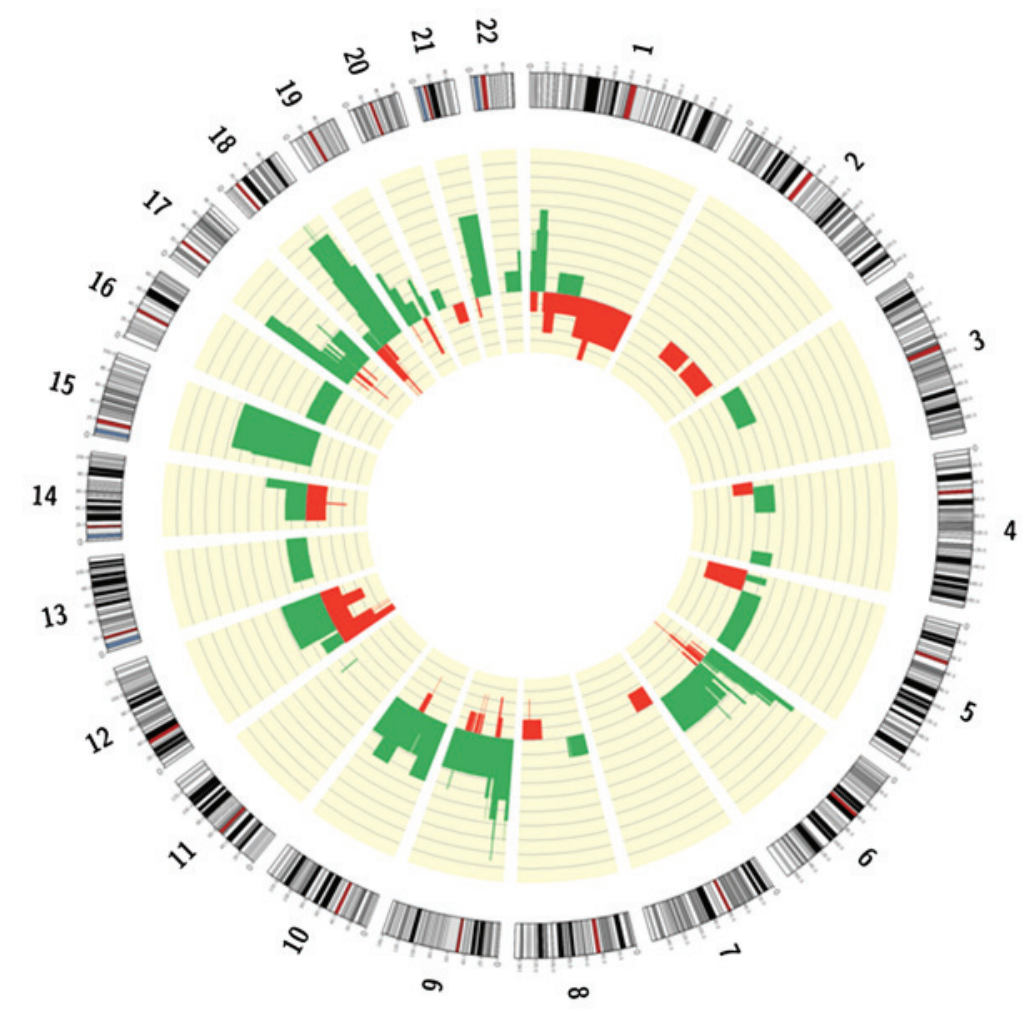

Figure 1. Macroscopic and cryptic cytogenetic alterations identified through single-nucleotide polymorphism array technology.

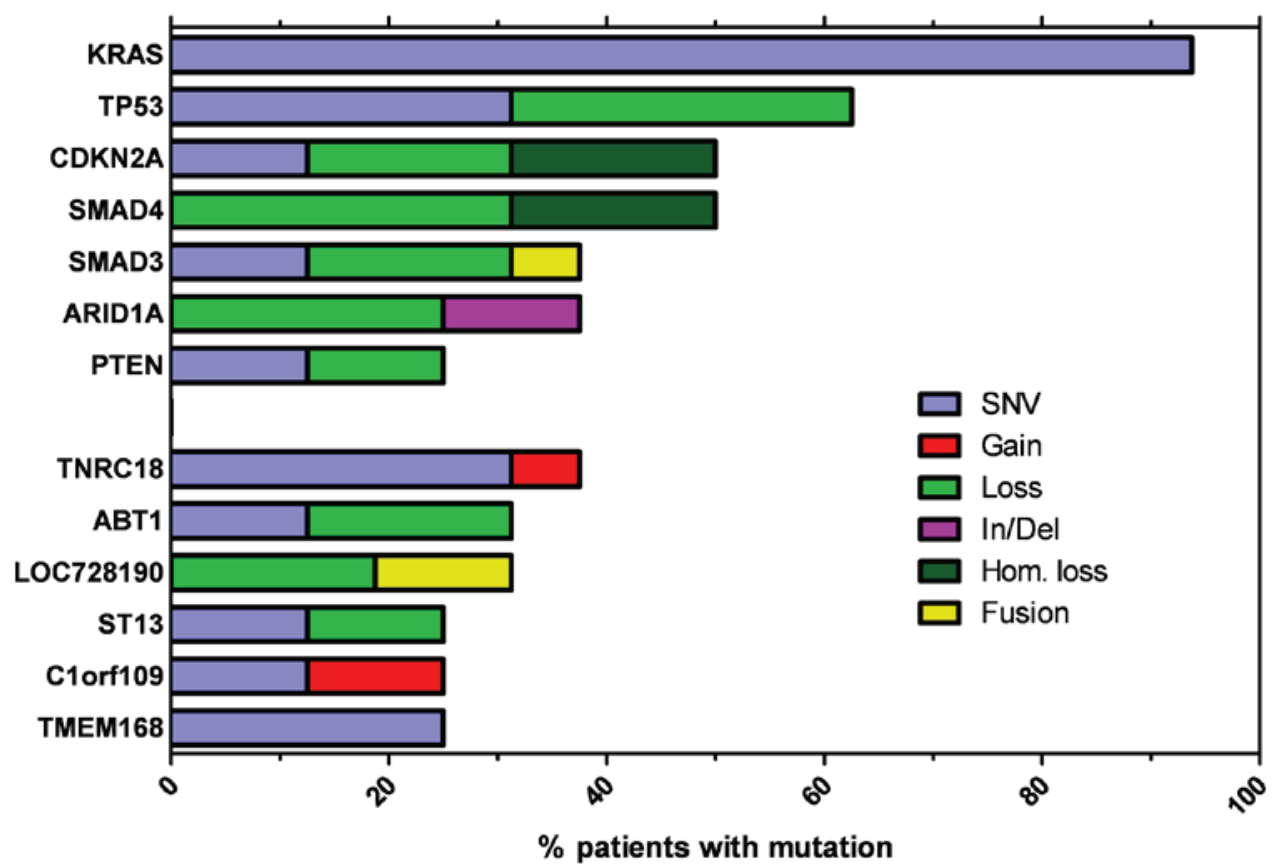

Figure 2. Most recurrent genes altered by point mutation, small In/Del and heterozygous or homozygous copy number loss. KRAS, Kirsten rat sarcoma viral oncogene homolog; TP53, tumor protein 53; CDKN2A, cyclin-dependent kinase inhibitor 2A; SMAD4, mothers against decapentaplegic homolog 4; ARID1A, AT-rich interactive domain-containing protein 1A; PTEN, phosphatase and tensin homolog; TNRC18, trinucleotide repeat containing 18; ABT1, activator of basal transcription 1; LOC728190, locus 728190; ST13, suppression of tumorigenicity 13; C1orf109, chromosome 1 open reading frame 109; TMEM168, transmembrane protein 168; SNV, single-nucleotide variant; In/Del, insertion or deletion; Hom. loss, loss of homozygosity.

The majority of single gene mutations in pancreatic cancer are grouped into common cellular pathways. Jones et al (11) identified 69 mutated gene sets in the majority of the 24 samples analyzed, of which 31 were grouped into 12 core signaling pathways. These pathways included KRAS, TGF- $\beta$, DNA damage control, apoptosis and regulation of $\mathrm{G}_{1} / \mathrm{S}$ cell cycle transition, involving KRAS, CDKN2A, TP53 and SMAD4. In addition, pathways including Hedgehog signaling, 
the homophilic cell adhesion pathway, integrin signaling, TGF- $\beta$ signaling, Wnt/Notch signaling, and regulation of the invasion pathway were identified (11).

The current study confirmed the high prevalence of KRAS, CDKN2A, TP53 and SMAD4 mutations. In particular, $93.7 \%$ of tumor samples exhibited somatic mutations activating KRAS and gene amplifications. Although the high KRAS mutation prevalence is an experimental design bias, as samples were included in the study only if a mutation in any RAS gene was observed, a previous study indicated a similar mutation frequency (12).

Notably, a G13D NRAS mutation was identified in the one case negative for KRAS. Ras proteins are GTPases with a high frequency of mutations in human tumors, however KRAS isoform mutations are prominent in pancreatic cancer compared with mutations of NRAS isoforms, which are more common in malignant melanomas (13).

KRAS is a key oncogene during the onset of pancreatic cancer however an effective KRAS inhibitor remains to be identified (14). SNP array technologies in the present study indicated amplifications at the chromosome arms harboring additional oncogenic genes such as GATA6, MYC and AKT2. In particular, AKT2 is involved in the phosphoinositide 3-kinase (PI3K) pathway, one of the feedback mechanisms through which KRAS maintains its levels of activity (15). Williams et al (16) observed that the combined use of mitogen-activated protein kinase (MAPK) and AKT inhibitors increased tumor sensitivity to radiation in a xenograft model of pancreatic cancer, while Diersch et al (15) demonstrated how PI3K inhibition reduced proliferation and tumor growth in a KPC mouse model of PDAC.

Therefore inhibition of PI3K or of additional pathways activated downstream of KRAS, such as MAPK signaling, may be an alternative way to target KRAS in PDAC (7).

The current study observed SMAD4 inactivation due to hetero- or homozygous focal loss of the SMAD4 locus (18q21.2) involved in the TGF- $\beta$ pathway. SMAD family proteins involved in the TGF- $\beta$ pathway were organized into two gene clusters, one at 18q21 chromosome (SMAD2, SMAD4 and SMAD7) and one at 15q21-22 (SMAD3 and SMAD6) (17).

TGF- $\beta$ receptor 1 activates SMAD2 and SMAD3, which bind to the common partner SMAD4, while SMAD6 and SMAD7 have an inhibitory role and block the phosphorylation of SMAD2 or SMAD3 (18). The SMAD complex regulates the transcription of several TGF- $\beta$-dependent genes following nuclear translocation that may have a context-dependent, tumor suppressive or progressive role (18). However, in the current study SMAD4 inactivation was observed at a reduced frequency than reported in a previous study (19).

Hezel et al (20) reported a $90 \%$ of loss of heterozygosity at the SMAD4 locus in PDAC, 50\% of which exhibited an additional inactivation of the remaining allele. In the current study, $50 \%$ of SMAD4 inactivation was observed to be due to hetero- and homozygous focal loss of the SMAD4 locus (18q21.2) in 62.5 and $37.5 \%$ of SMAD4 inactivation samples, respectively. Notably, patients with homozygous loss of SMAD4 (37.5\% of SMAD4 mutation cases) exhibited a poorer prognosis and a more aggressive disease. Leung et al (21) demonstrated that SMAD4 expression suppressed PDAC metastasis in their orthotopic xenograft model and Blackford et al (22) reported that SMAD4 loss is associated with a worsened PDAC prognosis. Therefore, SMAD4 is suggested as a potential prognostic biomarker, which may aid in development of therapeutic strategies (23). However, the current study identified cases negative for SMAD4 mutations with SMAD3 inactivation due to point mutation, gene fusion or allelic loss, with a possible role in pancreatic carcinogenesis in $37.5 \%$.

The present study confirmed the important role in pancreatic carcinogenesis of the oncosuppressor genes TP53 and CDKN2A, however in addition PTEN inactivation was observed in $25 \%$ of samples and more frequently in advanced patients (4/8) compared with early stage subjects (0/7). PTEN, a negative regulator of the PI3K pathway, may be involved in the gain of metastatic potential of pancreatic tumor cells in the absence of SMAD4. Garcia-Carracedo et al (24) investigated the role of PI3K signaling dysregulation in intraductal papillary mucinous neoplasm (IPMN), and loss of heterozygosity status at PTEN was observed in $35.7 \%$ of the IPMN cases analyzed. This indicated that PTEN downregulation was associated with a poor prognosis in patients with IPMN (24).

The current study demonstrated a high frequency of alterations in genes involved in DNA damage repair and chromatin remodeling. This highlights the relevance of the impaired DNA damage response in pancreatic carcinogenesis, in agreement with previous studies $(8,25,26)$. Dong et al (27) reported that mismatch repair gene variants may affect susceptibility to pancreatic cancer, observing that 28 SNPs were associated with altered pancreatic cancer risk $(\mathrm{P}<0.05)$.

In addition, the present study demonstrated ARID1A alterations in 6 of the 16 patients due to heterozygous focal genomic deletions or small frameshift In/Dels. ARID1A is a gene involved in DNA repair through the ATP-dependent induction of chromatin migration and dissociation, and its loss is frequently observed in ovarian clear cell adenocarcinoma and endometrioid adenocarcinoma (28). A previous study indicated that ARID1A loss is associated with reduced disease-free survival and chemoresistance in ovarian clear cell adenocarcinoma, however ARID1A inactivation has been identified in different tumor types including pancreatic cancer (29-31).

Additionally, the current study observed mutations in genes with no known function, the most common being detected at trinucleotide repeat containing 18, locus 728190 , poliovirus receptor related immunoglobulin domain, SH3-domain binding protein 2, transmembrane protein 168, DEAD box protein 60 and NHL repeat containing 2. Further studies are required to investigate the function and role of these gene mutations in pancreatic carcinogenesis.

In conclusion, the present study confirmed the tumoral heterogeneity of PDAC and identified known mutations in genes involved in RAS signaling, the p53 pathway and TGF- $\beta$ signaling, including SMAD4 and SMAD3 mutations. In addition, an emerging role for PTEN and ARID1A was identified and the importance of impaired DNA damage repair in creating the genetic instability responsible for cancer progression was emphasized. 


\section{Acknowledgements}

The present study was supported by PRIN 2009 New Therapeutic Strategies in Pancreatic Cancer and the Programma di Ricerca Regione-Università, Regione Emilia Romagna, bando Giovani Ricercatori 'Alessandro Liberati' 2013 to SV (no. PRUA1GR-2013-00000038). The authors would like to thank the Interdepartmental Center of Cancer Research for the technical support.

\section{References}

1. Siegel R, Naishadham D and Jemal A: Cancer statistics, 2013. CA Cancer J Clin 63: 11-30, 2013.

2. Conroy T, Desseigne F, Ychou M, Bouché O, Guimbaud R, Bécouarn Y, Adenis A, Raoul JL, Gourgou-Bourgade S, de la Fouchardière $\mathrm{C}$, et al: FOLFIRINOX versus gemcitabine for metastatic pancreatic cancer. N Engl J Med 364: 1817-1825, 2011.

3. Von Hoff DD, Ervin T, Arena FP, Chiorean EG, Infante J, Moore M, Seay T, Tjulandin SA, Ma WW, Saleh MN, et al: Increased survival in pancreatic cancer with nab-paclitaxel plus gemcitabine. N Engl J Med 369: 1691-1703, 2013

4. Di Marco M, Di Cicilia R, Macchini M, Nobili E, Vecchiarelli S, Brandi G and Biasco G: Metastatic pancreatic cancer: Is gemcitabine still the best standard treatment? (Review). Oncol Rep 23: 1183-1192, 2010.

5. Meyerson M, Gabriel S and Getz G: Advances in understanding cancer genomes through second-generation sequencing. Nat Rev Genet 11: 685-696, 2010

6. Feig C, Gopinathan A, Neesse A, Chan DS, Cook N and Tuveson DA: The pancreas cancer microenvironment. Clin Cancer Res 18: 4266-4276, 2012.

7. Collins MA and Pasca di Magliano M: Kras as a key oncogene and therapeutic target in pancreatic cancer. Front Physiol 4: 407, 2014.

8. Tan XG, Yang ZL, Yang LP and Miao XY: Expression of DNA-repair proteins and their significance in pancreatic cancer and non-cancerous pancreatic tissues of Sprague-Dawley rats. World J Surg Oncol 12: 32, 2014.

9. Hruban RH, Goggins M, Parsons J and Kern SE: Progression model for pancreatic cancer. Clin Cancer Res 6: 2969-2972, 2000

10. Iacobuzio-Donahue CA, Velculescu VE, Wolfgang CL and Hruban RH: Genetic basis of pancreas cancer development and progression: insights from whole-exome and whole-genome sequencing. Clin Cancer Res 18: 4257-4265, 2012.

11. Jones S, Zhang X, Parsons DW, Lin JC, Leary RJ, Angenendt P, Mankoo P, Carter H, Kamiyama H, Jimeno A, et al: Core signaling pathways in human pancreatic cancers revealed by global genomic analyses. Science 321: 1801-1806, 2008.

12. Yachida S, White CM, Naito Y, Zhong Y, Brosnan JA, Macgregor-Das AM, Morgan RA, Saunders T, Laheru DA, Herman JM, et al: Clinical significance of the genetic landscape of pancreatic cancer and implications for identification of potential long-term survivors. Clin Cancer Res 18: 6339-6347, 2012.

13. Fernández-Medarde A and Santos E: Ras in cancer and developmental diseases. Genes Cancer 2: 344-358, 2011.

14. Takashima A and Faller DV: Targeting the RAS oncogene. Expert Opin Ther Targets. 17: 507-531, 2013.

15. Diersch S, Wenzel P, Szameitat M, Eser P, Paul MC, Seidler B, Eser S, Messer M, Reichert M, Pagel P, et al: Efemp1 and p27(Kip1) modulate responsiveness of pancreatic cancer cells towards a dual PI3K/mTOR inhibitor in preclinical models. Oncotarget 4: 277-288, 2013.
16. Williams TM, Flecha AR, Keller P, Ram A, Karnak D, Galbán S, Galbán CJ, Ross BD, Lawrence TS, Rehemtulla A and Sebolt-Leopold J: Cotargeting MAPK and PI3K signaling with concurrent radiotherapy as a strategy for the treatment of pancreatic cancer. Mol Cancer Ther 11: 1193-1202, 2012.

17. Massagué J: TGFbeta in Cancer. Cell 134: 215-230, 2008.

18. Javle M, Li Y, Tan D, Dong X, Chang P, Kar S and Li D: Biomarkers of TGF- $\beta$ signaling pathway and prognosis of pancreatic cancer. PLoS One 9: e85942, 2014.

19. Chow JY, Dong H, Quach KT, Van Nguyen PN, Chen K and Carethers JM: TGF-beta mediates PTEN suppression and cell motility through calcium-dependent PKC-alpha activation in pancreatic cancer cells. Am J Physiol Gastrointest Liver Physiol 294: G899-G905, 2008.

20. Hezel AF, Kimmelman AC, Stanger BZ, Bardeesy N and Depinho RA: Genetics and biology of pancreatic ductal adenocarcinoma. Genes Dev 20: 1218-1249, 2006.

21. Leung L, Radulovich N, Zhu CQ, Wang D, To C, Ibrahimov E and Tsao MS: Loss of canonical Smad4 signaling promotes KRAS driven malignant transformation of human pancreatic duct epithelial cells and metastasis. PLoS One 8: e84366, 2013.

22. Blackford A, Serrano OK, Wolfgang CL, Parmigiani G, Jones S, Zhang X, Parsons DW, Lin JC, Leary RJ, Eshleman JR, et al: SMAD4 gene mutations are associated with poor prognosis in pancreatic cancer. Clin Cancer Res 15: 4674-4679, 2009.

23. Oshima M, Okano K, Muraki S, Haba R, Maeba T, Suzuki Y and Yachida S: Immunohistochemically detected expression of 3 major genes (CDKN2A/p16, TP53, and SMAD4/DPC4) strongly predicts survival in patients with resectable pancreatic cancer. Ann Surg 258: 336-346, 2013.

24. Garcia-Carracedo D, Turk AT, Fine SA, Akhavan N, Tweel BC, Parsons R, Chabot JA, Allendorf JD, Genkinger JM, Remotti HE and Su GH: Loss of PTEN expression is associated with poor prognosis in patients with intraductal papillary mucinous neoplasms of the pancreas. Clin Cancer Res 19: 6830-6841, 2013.

25. Lovejoy CA, Li W, Reisenweber S, Thongthip S, Bruno J, de Lange T, De S, Petrini JH, Sung PA, Jasin M, et al; ALT Starr Cancer Consortium: Loss of ATRX, genome instability, and an altered DNA damage response are hallmarks of the alternative lengthening of telomeres pathway. PLoS Genet 8: e1002772, 2012.

26. Waddell N, Pajic M, Patch AM, Chang DK, Kassahn KS, Bailey P, Johns AL, Miller D, Nones K, Quek K, et al; Australian Pancreatic Cancer Genome Initiative: Whole genomes redefine the mutational landscape of pancreatic cancer. Nature 518: 495-501, 2015.

27. Dong X, Li Y, Hess KR, Abbruzzese JL and Li D: DNA mismatch repair gene polymorphisms affect survival in pancreatic cancer. Oncologist 16: 61-70, 2011.

28. Hargreaves DC and Crabtree GR: ATP-dependent chromatin remodeling: Genetics, genomics and mechanisms. Cell Res 21: 396-420, 2011.

29. Katagiri A, Nakayama K, Rahman MT, Rahman M, Katagiri H, Nakayama N, Ishikawa M, Ishibashi T, Iida K, Kobayashi $\mathrm{H}$, et al: Loss of ARID1A expression is related to shorter progression-free survival and chemoresistance in ovarian clear cell carcinoma. Mod Pathol 25: 282-288, 2012.

30. Biankin AV, Waddell N, Kassahn KS, Gingras MC, Muthuswamy LB, Johns AL, Miller DK, Wilson PJ, Patch AM, Wu J, et al; Australian Pancreatic Cancer Genome Initiative: Pancreatic cancer genomes reveal aberrations in axon guidance pathway genes. Nature 491: 399-405, 2012.

31. Jones S, Li M, Parsons DW, Zhang X, Wesseling J, Kristel P, Schmidt MK, MarkowitzS, Yan H, Bigner D, et al: Somatic mutations in the chromatin remodeling gene ARID1A occur in several tumor types. Hum Mutat 33: 100-103, 2012. 